\title{
Numerical Implementation of Stochastic Operational Matrix Driven by a Fractional Brownian Motion for Solving a Stochastic Differential Equation
}

\author{
R. Ezzati, M. Khodabin, and Z. Sadati \\ Department of Mathematics, Karaj Branch, Islamic Azad University, Karaj, Iran \\ Correspondence should be addressed to R. Ezzati; ezati@kiau.ac.ir
}

Received 16 November 2013; Revised 20 January 2014; Accepted 20 January 2014; Published 11 March 2014

Academic Editor: Hamid Reza Karimi

Copyright (C) 2014 R. Ezzati et al. This is an open access article distributed under the Creative Commons Attribution License, which permits unrestricted use, distribution, and reproduction in any medium, provided the original work is properly cited.

An efficient method to determine a numerical solution of a stochastic differential equation (SDE) driven by fractional Brownian motion (FBM) with Hurst parameter $H \in(1 / 2,1)$ and $n$ independent one-dimensional standard Brownian motion (SBM) is proposed. The method is stated via a stochastic operational matrix based on the block pulse functions (BPFs). With using this approach, the SDE is reduced to a stochastic linear system of $m$ equations and $m$ unknowns. Then, the error analysis is demonstrated by some theorems and defnitions. Finally, the numerical examples demonstrate applicability and accuracy of this method.

\section{Introduction}

In many fields of science and engineering, there are a large number of problems which are intrinsically involving stochastic excitations of a Gaussian white noise type. Having in mind a Gaussian white noise mathematically described as a formal derivative of a Brownian motion process, all such problems are mathematically modeled by stochastic differential equations. Most of them cannot be solved analytically, so it is important to provide their numerical solutions. There has been a growing interest in numerical solutions of stochastic differential equations for the last years [1-10].

In the presented work, we consider SDE as follows:

$$
\begin{gathered}
d x(s)=k(s, t) x(s) d s+\sum_{i=1}^{n} t i(s, t) x(s) d B_{i}(s) \\
+r(s, t) x(s) d B_{s}^{H}, \quad s \in(0, T), T<1, \\
x(0)=x_{0},
\end{gathered}
$$

or

$$
\begin{aligned}
x(t)= & x_{0}+\int_{0}^{t} k(s, t) x(s) d s \\
& +\sum_{i=1}^{n} \int_{0}^{t} t i(s, t) x(s) d B_{i}(s) \\
& +\int_{0}^{t} r(s, t) x(s) d B_{s}^{H}, \quad t \in(0, T), T<1,
\end{aligned}
$$

where $B_{s}^{H}$ denotes the FBM with Hurst parameter $H \in$ $((1 / 2), 1)$ on probability space $(\Omega, F, P)$ and $B_{i}(s)(i=$ $1,2, \ldots, n)$ is $n$ independent one-dimensional SBM defined on the same probability space. Also, $k(s, t), t_{i}(s, t):(0, T) \times$ $(0, T) \rightarrow \mathbb{R}(i=1,2, \ldots, n)$ and $x(t)$ is the stochastic process of unknown on the probability space.

Investigations concerning the SDE driven by the FBM have been done by Zähle [11], Coutin [12], Decreusefond and Üstünel [13], Nualart [4, 14], Lisei and Soós [15], and other authors. Also, there exist several ways for solving it, pathwise and related techniques, Dirichlet forms, Euler approximations, Malliavin calculus, and Skorohod integral 
[1, 4, 15-17]; almost all methods have very poor numerical convergence.

It is important to find approximate solutions of the stochastic equations driven by the FBM, since these equations cannot be solved analytically in most cases and have many applications in models arising in physics, telecommunication networks, and finance [18]. Also, we cannot use from the classical Ito theory for their stochastic calculus, since these processes are not Markovian and semimartingale. Hence, in this work, we implement the stochastic operational matrix based on the BPFs for solving (2). The benefits of this method are lower cost of setting up the system of equations; moreover, the computational cost of operations is low. Also, convergence of this method is faster than other methods. These advantages make the method easier to apply.

The rest of the paper is organized as follows. In Section 2, some essential definitions and the following assumptions on the coefficients of (2) are stated. Also, the necessary properties of the block pulse functions (BPFs) are introduced. In Section 3, first a theorem is proved; then (2) is reduced to a stochastic linear system by using the properties of the BPFs. In Section 4, the error analysis is demonstrated. Efficiency of this method and good reasonable degree of accuracy are confirmed by some numerical examples, in Section 5. Finally, in Section 6, a brief conclusion is given.

\section{Preliminaries}

Definition 1. Let be the step $r(t)=\sum_{j=1}^{m-1} d_{j} \chi_{\left[t_{j}, t_{j+1}(t)\right]}$ function and $\chi$ denotes the characteristic function on $[0, p], d_{j} \in \mathbb{R}$, and $0=t_{1}<t_{2}<\cdots<t_{m}=p$. Then, the wiener integral with respect to the FBM is defined as

$$
\int_{0}^{p} r(t) d B_{t}^{H}=\sum_{j=1}^{m-1} d_{j}\left(B_{t_{j+1}}^{H}-B_{t_{j}}^{H}\right)
$$

where $H \in((1 / 2), 1)$ and $p>0$ (see [19]).

Definition 2. Let $\nu=v[\alpha, \lambda]$ denote the class of function $h$ on $[\alpha, \lambda] \times \Omega$ such that

(1) the function $h$ is $\beta \times F$ measurable;

(2) the function $h$ is adapted to $\left\{F_{t}\right\}_{t \geq 0}$;

(3) $\int_{\alpha}^{\lambda} \int_{\alpha}^{s} E\left[h^{2}(s)\right]|s-t| d t d s<\infty$ and $s, t \in[\alpha, \lambda]$.

Let us consider the following assumptions on the coefficients.

$\left(\mathrm{A}_{1}\right)(r(s, t) x(s)$ is differentiable in $x(s)$ and there exist constants $\alpha, \beta \leq 1$ and $K_{1}, K_{2}, K_{3}>0$ such that

$$
\begin{aligned}
& |r(s, t) x(s)-r(s, t) y(s)| \\
& \quad \leq K_{1}|x(s)-y(s)| \quad \text { (Lipschitz continuity) }
\end{aligned}
$$

$$
\begin{aligned}
& \left|\partial_{x} r(s, t) x(s)-\partial_{x} r(s, t) y(s)\right| \\
& \quad \leq K_{2}|x(s)-y(s)|^{\alpha} \quad \text { (Holder continuity), } \\
& |r(s, t) x(s)-r(u, t) x(s)|+\left|\partial_{x} r(s, t) x(s)-\partial_{x} r(u, t) x(s)\right| \\
& \quad \leq K_{3}|s-u|^{\beta} .
\end{aligned}
$$

$\left(\mathrm{A}_{2}\right)$ There exist constants $K_{4 i}, K_{5 i}>0(i=1, \ldots, n)$ such that

$$
\begin{aligned}
& |t i(s, t) x(s)-t i(s, t) y(s)| \\
& \leq K_{4 i}|x(s)-y(s)| \quad(\text { Lipschitz continuity), } \\
& |t i(s, t) x(s)| \leq K_{5 i}(1+|x(s)|) \quad \text { (Linear growth). }
\end{aligned}
$$

$\left(\mathrm{A}_{3}\right)$ There exist constants $K_{6}, K_{7}>0(i=1, \ldots, n)$ such that

$$
\begin{aligned}
& |k(s, t) x(s)-k(s, t) y(s)| \\
& \quad \leq K_{6}|x(s)-y(s)| \quad(\text { Lipschitz continuity), } \\
& |k(s, t) x(s)| \leq K_{7}(1+|x(s)|) \quad \text { (Linear growth), }
\end{aligned}
$$

for all $t, s, u \in(0, T)$.

Theorem 3. Let $k(s, t) x(s)$, ti $(s, t) x(s)$ and $r(s, t) x(s)$ hold in condition $\left(A_{1}\right),\left(A_{2}\right),\left(A_{3}\right)$, and $H<\{(1 / 2),(\alpha /(\alpha+1)), \beta\}$. Then, there exists a unique solution for (2).

Proof. See [18].

Now, we review the main properties of the BPFs which are necessary for this paper. Note that the BPFs are discussed in $[7,8]$.

(1) A function $p(x) \in L^{2}([0, T))$ is approximated by using properties of the BPFs as

$$
p(x) \approx \widehat{p}(x)=P^{T} \Psi(x)=\Psi^{T}(x) P,
$$

where

$$
\Psi(x)=\left(\Psi_{1}(x), \Psi_{2}(x), \ldots, \Psi_{i}(x), \ldots, \Psi_{m}(x)\right)^{T},
$$

with

$$
\Psi_{i}(x)= \begin{cases}1 & (i-1) \frac{T}{m} \leq x<i \frac{T}{m}, i=1, \ldots, m, \\ 0 & \text { otherwise, }\end{cases}
$$

where $\Psi_{i}(x)$ denotes the BPFs and

$$
P=\left(p_{1}, p_{2}, \ldots, p_{i}, \ldots, p_{m}\right)^{T}
$$


with

$$
p_{i}=\frac{m}{T} \int_{0}^{T} p(x) \Psi_{i}(x) d x
$$

(2) A function $p(x, y) \in L^{2}([0, T) \times[0, T))$ is approximated as follows:

$$
p(x, y) \approx \widehat{p}(x, y)=\Psi^{T}(x) P \Psi(y)=\Psi^{T}(y) P^{T} \Psi(x),
$$

where

$$
\begin{gathered}
P=\left(p_{i j}\right)_{m \times m}, \\
p_{i j}=\frac{m^{2}}{T^{2}} \iint_{0}^{T} p(x, y) \Psi_{i}(x) \Psi_{j}(y) d x d y, \\
(3) \text { Consider } \\
\Psi(x) \Psi^{T}(x) \\
=\left(\begin{array}{ccccc}
\Psi_{1}(x) & 0 & 0 & \ldots & 0 \\
0 & \Psi_{2}(x) & 0 & \ldots & 0 \\
0 & 0 & \Psi_{3}(x) & \ldots & 0 \\
\vdots & \vdots & \vdots & \ddots & \vdots \\
0 & 0 & 0 & \ldots & \Psi_{m}(x)
\end{array}\right)_{m \times m} .
\end{gathered}
$$

(14) where
(4) $\Psi(x) \Psi^{T}(x) L=\widehat{L} \Psi(x)$, where

$$
L=\left(l_{11}, l_{21}, \ldots, l_{m 1}\right)^{T},
$$

$$
\widehat{L}=\left(\begin{array}{ccccc}
l_{11} & 0 & 0 & \cdots & 0 \\
0 & l_{21} & 0 & \cdots & 0 \\
0 & 0 & l_{31} & \cdots & 0 \\
\vdots & \vdots & \vdots & \ddots & \vdots \\
0 & 0 & 0 & \cdots & l_{m 1}
\end{array}\right)_{m \times m} .
$$

(5) In [8], it is proved that

$$
\left(\int_{0}^{t} \Psi(x) \Psi^{T}(x) d x\right) A \Psi(t)=R \Psi(t),
$$

where

$$
R=\frac{T}{2 m}\left(\begin{array}{ccccc}
a_{11} & 2 a_{12} & 2 a_{13} & \cdots & 2 a_{1 m} \\
0 & a_{22} & 2 a_{23} & \cdots & 2 a_{2 m} \\
0 & 0 & a_{33} & \cdots & 2 a_{3 m} \\
\vdots & \vdots & \vdots & \ddots & \vdots \\
0 & 0 & 0 & \cdots & a_{m m}
\end{array}\right)_{m \times m} .
$$

(6) In [8], it is proved that

$$
\left(\int_{0}^{t} \Psi(x) \Psi^{T}(x) d B(x)\right) A \Psi(t)=E \Phi(t),
$$

$$
\begin{aligned}
& E=\left(\begin{array}{ccccc}
a_{11} B\left(\frac{h}{2}\right) & a_{12} B(h) & a_{13} B(h) & \cdots & a_{1 m} B(h) \\
0 & a_{22}\left(B\left(\frac{3 h}{2}\right)-B(h)\right) & a_{23}(B(2 h)-B(h)) & \cdots & a_{2 m}(B(2 h)-B(h)) \\
0 & 0 & a_{33}\left(B\left(\frac{5 h}{2}\right)-B(2 h)\right) & \cdots & a_{3 m}(B(3 h)-B(2 h)) \\
\vdots & \vdots & \vdots & \ddots & \vdots \\
0 & 0 & 0 & \cdots & a_{m m}\left(B\left(\frac{(2 m-1) h}{2}\right)-B((m-1) h)\right)
\end{array}\right)_{m \times m} \\
& h=\frac{T}{m} .
\end{aligned}
$$




\section{Solving the SDE Driven by FBM and $n$ Independent One-Dimensional SBM}

Theorem 4. Let $\Psi(t)$ denote the BPFs, $h=(T / m)$, and $R=$ $\left(r_{i j}\right)_{m \times m}, i, j=1, \ldots, m$; then

$$
\left(\int_{0}^{t} \Psi(s) \Psi^{T}(s) d B_{s}^{H}\right) R \Psi(t) \approx B \Psi(t), \quad t \in(0, T),
$$

where

$$
B=\left(\begin{array}{cccc}
r_{11} B_{h / 2}^{H} & r_{12} B_{h}^{H} & \cdots & r_{1 m} B_{h}^{H} \\
0 & r_{22}\left(B_{3 h / 2}^{H}-B_{h}^{H}\right) & \cdots & r_{2 m}\left(B_{2 h}^{H}-B_{h}^{H}\right) \\
\vdots & \vdots & \ddots & \vdots \\
0 & 0 & \cdots & r_{m m}\left(B_{((2 m-1) h / 2)}^{H}-B_{(m-1) h}^{H}\right)
\end{array}\right)_{m \times m}
$$

Proof. First, we compute stochastic operational matrix driven by the FBM based on the BPFs as follows.

(A1) If $0 \leq t<(i-1) h$, then

$$
\int_{0}^{t} \Psi_{i}(s) d B_{s}^{H}=0 .
$$

(A2) If $(i-1) h \leq t<i h$, the function $\Psi_{i}(s)$ is defined as

$$
\Psi_{i}(s)=\sum_{k=1}^{m+1} f_{i k} \chi_{\left[s_{k-1}, s_{k}\right)}(s), \quad i=1, \ldots, m,
$$

where $\chi$ denotes the characteristic function and $0=s_{0}<$ $s_{1}<\cdots<s_{i-1} \leq s_{i}=t<s_{i+1}<\cdots<s_{m+1}$, where $s_{k}=k h$ if $k=0,1, \ldots, i-1$ and $s_{k}=(k-1) h$ if $k=i+1, \ldots, m+1$. Also,

$$
f_{i k}= \begin{cases}1 & k=i \vee k=i+1 \\ 0 & \text { otherwise }\end{cases}
$$

Now, for computation $\int_{0}^{t} \Psi_{i}(s) d B_{s}^{H_{i}}(s \in[0, t])$, we can write

$$
\Psi_{i}(s)=\sum_{k=1}^{i} f_{i k} \chi_{\left[s_{k-1}, s_{k}\right)}(s), \quad i=1, \ldots, m .
$$

Then by using Definition 1, we obtain

$$
\begin{aligned}
\int_{0}^{t} \Psi_{i}(s) d B_{s}^{H} & =\sum_{k=1}^{i} f_{i k}\left(B_{s_{k}}^{H}-B_{s_{k-1}}^{H}\right) \\
& =B_{t}^{H}-B_{(i-1) h}^{H}, \quad i=1,2, \ldots, m .
\end{aligned}
$$

(A3) If $i h \leq t<T$, then

$$
\Psi_{i}(s)=\sum_{k=1}^{m+1} c_{i k} \chi_{\left[s_{k-1}, s_{k}\right)}(s), \quad i=1, \ldots, m,
$$

where $0=s_{0}<s_{1}<\cdots<s_{i} \leq s_{i+1}=t<s_{i+2}<\cdots<s_{m+1}$, $s_{k}=k h$ if $k=0,1, \ldots, i, s_{k}=(k-1) h$ if $k=i+2, \ldots, m+1$, and

$$
c_{i k}= \begin{cases}1 & k=i \\ 0 & k \neq i\end{cases}
$$

For computation $\int_{0}^{t} \Psi_{i}(s) d B_{s}^{H_{i}}(s \in[0, t])$, we can write

$$
\Psi_{i}(s)=\sum_{k=1}^{i+1} c_{i k} \chi_{\left[s_{k-1}, s_{k}\right)}(s), \quad i=1, \ldots, m,
$$

so, we get

$$
\begin{aligned}
\int_{0}^{t} \Psi_{i}(s) d B_{s}^{H} & =\sum_{k=1}^{i+1} c_{i k}\left(B_{s_{k}}^{H}-B_{s_{k-1}}^{H}\right) \\
& =B_{i h}^{H}-B_{(i-1) h}^{H}, \quad i=1,2, \ldots, m .
\end{aligned}
$$

From (A1), (A2), and (A3), we get

$$
\int_{0}^{t} \Psi_{i}(s) d B_{s}^{H}= \begin{cases}0 & 0 \leq t<(i-1) h, \\ B_{t}^{H}-B_{(i-1) h}^{H} & (i-1) h \leq t<i h, \\ B_{i h}^{H}-B_{(i-1) h}^{H} & i h \leq t<T .\end{cases}
$$

Furthermore, we suppose that

$$
B_{t}^{H}-B_{(i-1) h}^{H} \approx B_{(i-0.5) h}^{H}-B_{(i-1) h}^{H}, \quad(i-1) h \leq t<i h,
$$

so, we can write

$$
\begin{aligned}
\int_{0}^{t} \Psi_{i}(s) d B_{s}^{H} \approx( & 0, \ldots, 0, B_{(i-0.5) h}^{H}-B_{(i-1) h}^{H}, \\
& \left.B_{i h}^{H}-B_{(i-1) h}^{H}, \ldots, B_{i h}^{H}-B_{(i-1) h}^{H}\right) \Psi(t) .
\end{aligned}
$$

Hence, by using the relation (33), we can write

$$
\int_{0}^{t} \Psi(s) d B_{s}^{H} \approx P_{H} \Psi(t)
$$


where

$$
P_{H}=\left(\begin{array}{ccccc}
B_{h / 2}^{H} & B_{h}^{H} & B_{h}^{H} & \cdots & B_{h}^{H} \\
0 & B_{3 h / 2}^{H}-B_{h}^{H} & B_{2 h}^{H}-B_{h}^{H} & \cdots & B_{2 h}^{H}-B_{h}^{H} \\
0 & 0 & B_{5 h / 2}^{H}-B_{2 h}^{H} & \cdots & B_{3 h}^{H}-B_{2 h}^{H} \\
\vdots & \vdots & \vdots & \ddots & \vdots \\
0 & 0 & 0 & \cdots & B_{((2 m-1) h / 2)}^{H}-B_{(m-1) h}^{H}
\end{array}\right)_{m \times m}
$$

Now, let $C_{R^{i}}$ be the $i$ th row of matrix $C_{R}=\operatorname{Diag}(R)$, let $P_{H}^{i}$ be the ith row of the matrix $P_{H}$, and let $R^{i}$ be the ith row of matrix $R$. We have

$$
\begin{aligned}
& \left(\int_{0}^{t} \Psi(s) \Psi^{T}(s) d B_{s}^{H}\right) R \Psi(t) \\
& \approx\left(\begin{array}{c}
P_{H}^{1} \Psi(t) R^{1} \Psi(t) \\
P_{H}^{2} \Psi(t) R^{2} \Psi(t) \\
\vdots \\
P_{H}^{m} \Psi(t) R^{m} \Psi(t)
\end{array}\right) \approx\left(\begin{array}{c}
P_{H}^{1} C_{R^{1}} \\
P_{H}^{2} C_{R^{2}} \\
\vdots \\
P_{H}^{m} C_{R^{m}}
\end{array}\right) \Psi(t) \\
& \approx B \Psi(t) \text {, }
\end{aligned}
$$

where $B$ is given by (21).

Let

$$
\begin{gathered}
x(t) \approx x^{T} \Psi(t)=\Psi^{T}(t) x, \\
x_{0} \approx x_{0}^{T} \Psi(t)=\Psi^{T}(t) x_{0}, \\
k(s, t) \approx \Psi^{T}(s) K \Psi(t)=\Psi^{T}(t) K^{T} \Psi(s), \\
r(s, t) \approx \Psi^{T}(s) R \Psi(t)=\Psi^{T}(t) R^{T} \Psi(s), \\
t i(s, t) \approx \Psi^{T}(s) T i \Psi(t) \\
=\Psi^{T}(t) T i^{T} \Psi(s), \quad i=1,2, \ldots, n,
\end{gathered}
$$

where $x$ and $x_{0}$ are the block pulse coefficients vector and $K$, $R$, and $T i, i=1, \ldots, n$, are the block pulse coefficients matrix.
By substituting the relation (37) in (2), we get

$$
\begin{aligned}
x^{T} \Psi(t) \approx & x_{0}^{T} \Psi(t)+x^{T} \int_{0}^{t} \Psi(s) \Psi^{T}(s) K \Psi(t) d s \\
& +\sum_{i=1}^{n} x^{T} \int_{0}^{t} \Psi(s) \Psi^{T}(s) T i \Psi(t) d B_{i}(s) \\
& +x^{T} \int_{0}^{t} \Psi(s) \Psi^{T}(s) R \Psi(t) d B_{s}^{H},
\end{aligned}
$$

or

$$
\begin{aligned}
x^{T} \Psi(t) \approx & x_{0}^{T} \Psi(t)+x^{T}\left(\int_{0}^{t} \Psi(s) \Psi^{T}(s) d s\right) K \Psi(t) \\
& +x^{T} \sum_{i=1}^{n}\left(\int_{0}^{t} \Psi(s) \Psi^{T}(s) d B_{i}(s)\right) T i \Psi(t)
\end{aligned}
$$

$$
+x^{T}\left(\int_{0}^{t} \Psi(s) \Psi^{T}(s) d B_{s}^{H}\right) R \Psi(t) .
$$

Therefore, by using properties of the BPFs and Theorem 4, we can write

$$
\begin{aligned}
x^{T} \Psi(t) \approx & x_{0}^{T} \Psi(t)+x^{T} A \Psi(t) \\
& +x^{T} \sum_{i=1}^{n} C_{i} \Psi(t)+x^{T} B \Psi(t),
\end{aligned}
$$

where

$$
A=\frac{T}{2 m}\left(\begin{array}{ccccc}
k_{11} & 2 k_{12} & 2 k_{13} & \cdots & 2 k_{1 m} \\
0 & k_{22} & 2 k_{23} & \cdots & 2 k_{2 m} \\
0 & 0 & k_{33} & \cdots & 2 k_{3 m} \\
\vdots & \vdots & \vdots & \ddots & \vdots \\
0 & 0 & 0 & \cdots & k_{m m}
\end{array}\right) \text {, }
$$




$$
\begin{aligned}
& k_{i j}=\frac{m^{2}}{T^{2}} \iint_{0}^{T} k(s, t) \Psi_{i}(s) \Psi_{j}(t) d s d t, \\
& i=1,2, \ldots, m, j=1,2, \ldots, m,
\end{aligned}
$$

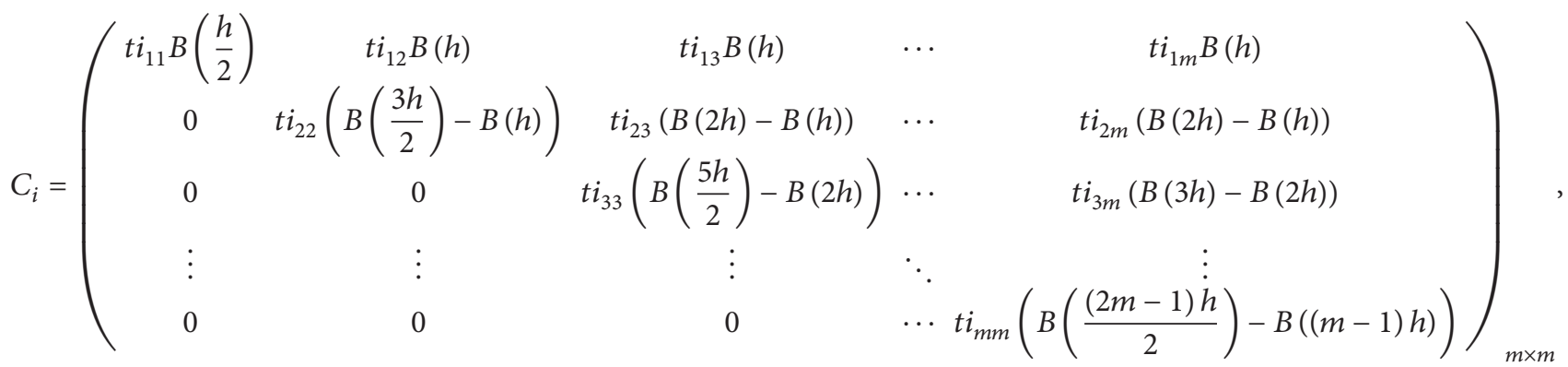

with

$$
\begin{gathered}
t i_{p q}=\frac{m^{2}}{T^{2}} \iint_{0}^{T} t i(s, t) \Psi_{p}(s) \Psi_{q}(t) d s d t, \\
p=1,2, \ldots, m, q=1,2, \ldots, m, \\
B=\left(\begin{array}{cccc}
r_{11} B_{h / 2}^{H} & r_{12} B_{h}^{H} & \cdots & r_{1 m} B_{h}^{H} \\
0 & r_{22}\left(B_{3 h / 2}^{H}-B_{h}^{H}\right) & \cdots & r_{2 m}\left(B_{2 h}^{H}-B_{h}^{H}\right) \\
\vdots & \vdots & \ddots & \vdots \\
0 & 0 & \cdots & r_{m m}\left(B_{((2 m-1) h / 2)}^{H}-B_{(m-1) h}^{H}\right)
\end{array}\right)_{m \times m},
\end{gathered}
$$

with

$$
\begin{aligned}
r_{i j}=\frac{m^{2}}{T^{2}} \iint_{0}^{T} r(s, t) \Psi_{i}(s) \Psi_{j}(t) d s d t, \\
\quad i=1,2, \ldots, m, j=1,2, \ldots, m .
\end{aligned}
$$

$$
=2 H(2 H-1)
$$

$$
\times \int_{\alpha}^{\beta} \int_{\alpha}^{y}(y-x)^{2 H-2} E\left[f^{2}(x)\right] d x d y .
$$

Now, with replacing $\approx$ by $=$, we have

$$
x^{T}\left(I-A-\sum_{i=1}^{n} C_{i}-B\right) \Psi(t)=x_{0}^{T} \Psi(t),
$$

or

$$
M x=x_{0},
$$

where $M=\left(I-A-\sum_{i=1}^{n} C_{i}-B\right)^{T}$. Clearly, (46) is the stochastic linear system of $m$ equations and $m$ unknowns.

\section{Error Analysis}

In [20], it is stated that if $f(t) \in v[\alpha, \beta]$ and $(1 / 2)<H<1$, then

$$
\begin{aligned}
& E\left[\left(\int_{\alpha}^{\beta} f(x) d B_{x}^{H}\right)^{2}\right] \\
& =\int_{\alpha}^{\beta} E\left[(f(x))^{2}\right](d x)^{2 H}
\end{aligned}
$$

Theorem 5. Let $r(s)$ be an arbitrary bounded function on $[0,1)$ and $e(s)=r(s)-\widehat{r}(s)$ such that $\widehat{r}(s)$ is the BPFs of $r(s)$. Then,

$$
|e(s)|^{2} \leq O\left(h^{2}\right), \quad 0 \leq s<1
$$

Proof. See [7].

Theorem 6. Let $r(x, y)$ be an arbitrary bounded function on $I=[0,1) \times[0,1)$ and $e(x, y)=r(x, y)-\widehat{r}(x, y)$ such that $\widehat{r}(x, y)$ is the BPFs of $r(x, y)$. Then,

$$
|e(x, y)|^{2} \leq O\left(h^{2}\right), \quad(x, y) \in I .
$$

Proof. See [7]. 


$$
\begin{aligned}
& \text { Let } \\
& \begin{aligned}
e(t)= & x(t)-\widehat{x}(t)=x_{0}-\widehat{x}_{0} \\
& +\int_{0}^{t}(k(s, t) x(s)-\widehat{k}(s, t) \hat{x}(s)) d s \\
& +\sum_{i=1}^{n} \int_{0}^{t}(t i(s, t) x(s)-\widehat{t i}(s, t) \widehat{x}(s)) d B_{i}(s) \\
& +\int_{0}^{t}(r(s, t) x(s)-\widehat{r}(s, t) \widehat{x}(s)) d B_{s}^{H},
\end{aligned}
\end{aligned}
$$

where $\widehat{x}(t)$ is the approximate solution of $x(t)$ defined in (46) and $\widehat{x}_{0}, \widehat{k}(s, t), \widehat{t i}(s, t)$, and $\widehat{r}(s, t)$ are approximated by using properties of the BPFs.

Theorem 7. Let $\widehat{x}(t)$ be the approximate solution of (2) which is the solution of (46), $\|x(t)\|^{2} \leq N,\|k(s, t)\|^{2} \leq l_{1},\|t i(s, t)\|^{2} \leq$ $l_{2 i}, i=1,2, \ldots, n$, and $\|r(s, t)\|^{2} \leq l_{3}$, for all $(s, t) \in I=[0,1) \times$ $[0,1)$. Then,

$$
\|x(t)-\widehat{x}(t)\|^{2} \leq O\left(h^{2}\right), \quad t \in[0,1),
$$

where $\|x\|=\left(E\left[x^{2}\right]\right)^{1 / 2}$.

Proof. Consider

$$
\begin{aligned}
e(t)= & x(t)-\widehat{x}(t)=x_{0}-\widehat{x}_{0} \\
& +\int_{0}^{t}(k(s, t) x(s)-\widehat{k}(s, t) \hat{x}(s)) d s \\
& +\sum_{i=1}^{n} \int_{0}^{t}(t i(s, t) x(s)-\widehat{t i}(s, t) \hat{x}(s)) d B_{i}(s) \\
& +\int_{0}^{t}(r(s, t) x(s)-\widehat{r}(s, t) \hat{x}(s)) d B_{s}^{H} ;
\end{aligned}
$$

by using $\left(\sum_{i=1}^{n+3} x_{i}\right)^{2} \leq(n+3)\left(\sum_{i=1}^{n+3} x_{i}^{2}\right)$, we can write

$$
\begin{aligned}
&\|x(t)-\widehat{x}(t)\|^{2} \\
& \leq(n+3)\left(\left\|x_{0}-\widehat{x}_{0}\right\|^{2}\right. \\
&+\left\|\int_{0}^{t}(k(s, t) x(s)-\widehat{k}(s, t) \widehat{x}(s)) d s\right\|^{2} \\
&+\sum_{i=1}^{n}\left\|\int_{0}^{t}(t i(s, t) x(s)-\widehat{t i}(s, t) \widehat{x}(s)) d B_{i}(s)\right\|^{2} \\
&\left.+\left\|\int_{0}^{t}(r(s, t) x(s)-\widehat{r}(s, t) \widehat{x}(s)) d B_{s}^{H}\right\|^{2}\right) .
\end{aligned}
$$

First, by using the relation (47), we can write

$$
\begin{gathered}
E\left[\left(\int_{0}^{t}(r(s, t) x(s)-\widehat{r}(s, t) \hat{x}(s)) d B_{s}^{H}\right)^{2}\right] \\
=\int_{0}^{t} E\left[(r(s, t) x(s)-\hat{r}(s, t) \hat{x}(s))^{2}\right](d s)^{2 H}
\end{gathered}
$$

$$
\begin{aligned}
= & 2 H(2 H-1) \\
& \times \int_{0}^{t} \int_{0}^{p}(p-s)^{2 H-2} \\
& \times E\left[(r(s, t) x(s)-\hat{r}(s, t) \hat{x}(s))^{2}\right] d s d p \\
= & 2 H(2 H-1) \\
& \times \int_{0}^{t} \int_{s}^{t}(p-s)^{2 H-2} \\
& \times E\left[(r(s, t) x(s)-\widehat{r}(s, t) \hat{x}(s))^{2}\right] d p d s \\
= & 2 H(2 H-1) \int_{0}^{t} E\left[(r(s, t) x(s)-\hat{r}(s, t) \hat{x}(s))^{2}\right] \\
& \times \int_{s}^{t}(p-s)^{2 H-2} d p d s \\
= & 2 H \int_{0}^{t} E\left[(r(s, t) x(s)-\widehat{r}(s, t) \hat{x}(s))^{2}\right](t-s)^{2 H-1} d s .
\end{aligned}
$$

Cleary, we have

$$
\begin{gathered}
0<s<t<1, \\
0<2 H-1<1,
\end{gathered}
$$

and consequently,

$$
0<(t-s)^{2 H-1}<1
$$

Hence,

$$
\begin{aligned}
& E\left[\left(\int_{0}^{t} r(s, t) x(s)-\widehat{r}(s, t) \hat{x}(s) d B_{s}^{H}\right)^{2}\right] \\
& \quad \leq 2 \int_{0}^{t} E\left[(r(s, t) x(s)-\widehat{r}(s, t) \hat{x}(s))^{2}\right] d s,
\end{aligned}
$$

or

$$
\begin{aligned}
& \left\|\int_{0}^{t}(r(s, t) x(s)-\widehat{r}(s, t) \hat{x}(s)) d B_{s}^{H}\right\|^{2} \\
& \quad \leq 2 \int_{0}^{t}\|r(s, t) x(s)-\widehat{r}(s, t) \hat{x}(s)\|^{2} d s .
\end{aligned}
$$

Now, by using the property of the Ito isometry for the SBM defined in [21] and $(x+y)^{2} \leq 2\left(x^{2}+y^{2}\right)$, we get

$$
\begin{aligned}
\|x(t)-\widehat{x}(t)\|^{2} & \\
\leq(n+3)( & \left\|x_{0}-\widehat{x}_{0}\right\|^{2} \\
& +\int_{0}^{t}\|k(s, t) x(s)-\widehat{k}(s, t) \hat{x}(s)\|^{2} d s \\
& +\sum_{i=1}^{n} \int_{0}^{t}\|t i(s, t) x(s)-\widehat{t i}(s, t) \hat{x}(s)\|^{2} d s \\
& \left.+2 \int_{0}^{t}\|r(s, t) x(s)-\widehat{r}(s, t) \hat{x}(s)\|^{2} d(s)\right)
\end{aligned}
$$


TABLE 1: Mean, standard deviation, and confidence interval for error mean $(T=0.25, H=2 / 3)$.

\begin{tabular}{lcccc}
\hline$t$ & $\bar{x}$ & $\bar{s}$ & \multicolumn{2}{c}{ \%95 confidence interval for mean } \\
Upper
\end{tabular}

TABLE 2: Mean, standard deviation, and confidence interval for numerical solution mean $(T=0.25, H=3 / 4)$.

\begin{tabular}{lcccc}
\hline$t$ & $\bar{x}$ & $\bar{s}$ & \multicolumn{2}{c}{ \%95 confidence interval for mean } \\
& & & $1.1235 \times 10^{-4}$ & Upper \\
\hline 0.05 & $1.9830 \times 10^{-4}$ & $1.3868 \times 10^{-4}$ & $8.196 \times 10^{-5}$ & $2.9425 \times 10^{-4}$ \\
0.1 & $1.8920 \times 10^{-4}$ & $1.7302 \times 10^{-4}$ & $2.5225 \times 10^{-4}$ & $4.9755 \times 10^{-4}$ \\
0.15 & $3.7490 \times 10^{-4}$ & $1.9789 \times 10^{-4}$ & $1.3312 \times 10^{-4}$ & $4.8568 \times 10^{-4}$ \\
0.2 & $3.0940 \times 10^{-4}$ & $2.8441 \times 10^{-4}$ & &
\end{tabular}

$$
\begin{aligned}
& \leq 2(n+3)\left(\left\|x_{0}-\widehat{x}_{0}\right\|^{2}\right. \\
& +2 \sum_{i=1}^{n} \int_{0}^{t}\left(\|t i(s, t)-\widehat{t i}(s, t)\|^{2}\right) \\
& +\int_{0}^{t} \| k(s, t)(x(s)-\widehat{x}(s)) \\
& +(k(s, t)-\widehat{k}(s, t)) \\
& \times(\widehat{x}(s)-x(s)+x(s)) \|^{2} d s \\
& \times\left(2 \left(\|x(s)-\widehat{x}(s)\|^{2}\right.\right. \\
& \left.\left.+\|x(s)\|^{2}\right)\right) d s \\
& +2 \int_{0}^{t}\|r(s, t)\|^{2}\|x(s)-\widehat{x}(s)\|^{2} d s \\
& +\sum_{i=1}^{n} \int_{0}^{t} \| t i(s, t)(x(s)-\widehat{x}(s)) \\
& +2 \int_{0}^{t}\left(\|r(s, t)-\widehat{r}(s, t)\|^{2}\right) \\
& +(t i(s, t)-\widehat{t i}(s, t)) \\
& \times(\widehat{x}(s)-x(s)+x(s)) \|^{2} d s \\
& \times\left(2 \left(\|x(s)-\widehat{x}(s)\|^{2}\right.\right. \\
& \left.\left.\left.+\|x(s)\|^{2}\right)\right) d s\right) \\
& +\int_{0}^{t} \| r(s, t)(x(s)-\widehat{x}(s)) \\
& +(r(s, t)-\widehat{r}(s, t)) \\
& \left.\times(\widehat{x}(s)-x(s)+x(s)) \|^{2} d s\right) \\
& \leq 8(n+3) \\
& \times\left(\left\|x_{0}-\widehat{x}_{0}\right\|^{2}\right. \\
& +\int_{0}^{t}\|k(s, t)\|^{2}\|x(s)-\widehat{x}(s)\|^{2} d s \\
& \leq 2(n+3)\left(\left\|x_{0}-\widehat{x}_{0}\right\|^{2}\right. \\
& +2 \int_{0}^{t}\|k(s, t)\|^{2} \\
& \times\|x(s)-\widehat{x}(s)\|^{2} d s \\
& +\int_{0}^{t}\left(\|k(s, t)-\widehat{k}(s, t)\|^{2}\right) \\
& \times\left(\|x(s)-\widehat{x}(s)\|^{2}+\|x(s)\|^{2}\right) d s \\
& +\sum_{i=1}^{n} \int_{0}^{t}\|t i(s, t)\|^{2}\|x(s)-\widehat{x}(s)\|^{2} d s \\
& +2 \int_{0}^{t}\left(\|k(s, t)-\widehat{k}(s, t)\|^{2}\right) \\
& +\sum_{i=1}^{n} \int_{0}^{t}\left(\|t i(s, t)-\widehat{t i}(s, t)\|^{2}\right) \\
& \times\left(2\left(\|x(s)-\widehat{x}(s)\|^{2}+\|x(s)\|^{2}\right)\right) d s \\
& \times\left(\|x(s)-\widehat{x}(s)\|^{2}+\|x(s)\|^{2}\right) d s \\
& +2 \sum_{i=1}^{n} \int_{0}^{t}\|t i(s, t)\|^{2}\|x(s)-\widehat{x}(s)\|^{2} d s \\
& +\int_{0}^{t}\|r(s, t)\|^{2}\|x(s)-\widehat{x}(s)\|^{2} d s
\end{aligned}
$$


TABLE 3: Mean, standard deviation, and confidence interval for numerical solution mean $(T=0.25, H=9 / 10)$.

\begin{tabular}{lcccc}
\hline$t$ & $\bar{x}$ & $\bar{s}$ & \multicolumn{2}{c}{ \%95 confidence interval for mean } \\
Upper
\end{tabular}

TABLE 4: Mean, standard deviation, and confidence interval for numerical solution mean $(T=0.25, H=2 / 3)$.

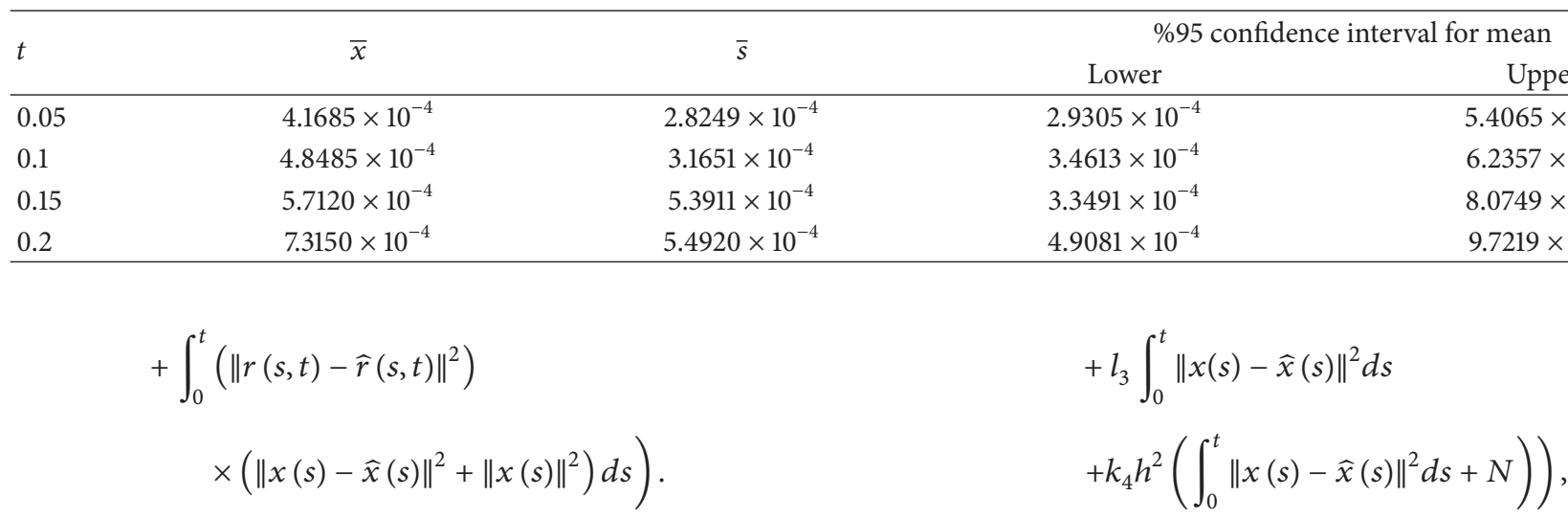

By using Theorems 5 and 6, we can write

$$
\begin{gathered}
\left\|x_{0}-\widehat{x}_{0}\right\|^{2} \leq k_{1} h^{2}, \\
\|k(s, t)-\widehat{k}(s, t)\|^{2} \leq k_{2} h^{2}, \\
\|t i(s, t)-\widehat{t i}(s, t)\|^{2} \leq k_{3 i} h^{2}, \quad i=1, \ldots, n, \\
\|r(s, t)-\widehat{r}(s, t)\|^{2} \leq k_{4} h^{2} .
\end{gathered}
$$

By substituting the relation (60) in (59), we get

$$
\begin{aligned}
\|x(t)-\widehat{x}(t)\|^{2} & \\
\leq & 8(n+3) \\
& \times\left(k_{1} h^{2}+l_{1} \int_{0}^{t}\|x(s)-\widehat{x}(s)\|^{2} d s\right. \\
& +k_{2} h^{2}\left(\int_{0}^{t}\|x(s)-\widehat{x}(s)\|^{2} d s+N\right) \\
& +\sum_{i=1}^{n} l_{2 i} \int_{0}^{t}\|x(s)-\widehat{x}(s)\|^{2} d s \\
& +\sum_{i=1}^{n} k_{3 i} h^{2}\left(\int_{0}^{t}\|x(s)-\widehat{x}(s)\|^{2} d s+N\right)
\end{aligned}
$$

or

$$
\|x(t)-\widehat{x}(t)\|^{2} \leq \mu+\lambda \int_{0}^{t}\|x(s)-\widehat{x}(s)\|^{2} d s
$$

where $\mu=8(n+3)\left(k_{1} h^{2}+k_{2} h^{2} N+\sum_{i=1}^{n} k_{3 i} h^{2} N+k_{4} h^{2} N\right)$ and $\lambda=8(n+3)\left(l_{1}+k_{2} h^{2}+\sum_{i=1}^{n} l_{2 i}+\sum_{i=1}^{n} k_{3 i} h^{2}+l_{3}+k_{4} h^{2}\right)$. If $f(s)=\|x(s)-\widehat{x}(s)\|^{2}$, we get

$$
f(t) \leq \mu+\lambda \int_{0}^{t} f(s) d s .
$$

Now, by using Gronwall inequality, we have

$$
f(t) \leq \mu\left(1+\lambda \int_{0}^{t} \exp (\lambda(t-s)) d s\right), \quad t \in[0,1),
$$

or

$$
\|x(t)-\widehat{x}(t)\|^{2} \leq O\left(h^{2}\right)
$$

\section{Numerical Examples}

The SDE driven by the FBM

$$
\begin{aligned}
S(t)= & S_{0}+\int_{0}^{t} \mu(s) S(s) d s \\
& +\sum_{i=1}^{n} \int_{0}^{t} \sigma i(s) S(s) d B_{i}(s) \\
& +\int_{0}^{t} \alpha(s) S(s) d B_{s}^{H}, \quad t \in(0, T),
\end{aligned}
$$


TABLE 5: Mean, standard deviation, and confidence interval for numerical solution mean $(T=0.25, H=3 / 4)$.

\begin{tabular}{lcccc}
\hline$t$ & $\bar{x}$ & $\bar{s}$ & \multicolumn{2}{c}{ \%95 confidence interval for mean } \\
Upper
\end{tabular}

TABLE 6: Mean, standard deviation, and confidence interval for numerical solution mean $(T=0.25, H=9 / 10)$.

\begin{tabular}{lcccc}
\hline$t$ & $\bar{x}$ & $\bar{s}$ & \multicolumn{2}{c}{ \%95 confidence interval for mean } \\
& & & $3.7299 \times 10^{-4}$ & Upper \\
\hline 0.05 & $4.9780 \times 10^{-4}$ & $2.0137 \times 10^{-4}$ & $4.2261 \times 10^{-4}$ \\
0.1 & $6.9850 \times 10^{-4}$ & $3.4267 \times 10^{-4}$ & $4.8611 \times 10^{-4}$ & $9.1089 \times 10^{-4}$ \\
0.15 & $7.7470 \times 10^{-4}$ & $5.1518 \times 10^{-4}$ & $4.5537 \times 10^{-4}$ & $1.0940 \times 10^{-3}$ \\
0.2 & $1.2516 \times 10^{-3}$ & $6.3210 \times 10^{-4}$ & $8.5982 \times 10^{-4}$ & $1.6434 \times 10^{-3}$ \\
\hline
\end{tabular}

is applied in modeling the price $S$ of a stock with various Hurst parameters (see [18]). Hence, we show applicability and accuracy of this method in two numerical examples.

Example 1. Let us consider a SDE

$$
\begin{gathered}
d x(s)=-\frac{1}{5} s^{2} x(s) d s-\frac{1}{10} x(s) d B_{s}^{H} \\
-\frac{1}{6} x(s) d B_{1}(s)-\frac{1}{30} x(s) d B_{2}(s), \\
s \in(0, T), T<1, \\
x(0)=\frac{1}{30},
\end{gathered}
$$

with the exact solution $x(t)=(1 / 30) \exp \left(-(1 / 10) B_{t}^{H}-\right.$ $(1 / 15) t^{3}-(1 / 200) \times t^{2 H}-(1 / 6) B_{1}(t)-(1 / 72) t-(1 / 30) B_{2}(t)-$ $(1 / 1800) t)$. The numerical results have been shown in Tables 1,2 , and 3 (with various Hurst parameters), where $\bar{x}$ and $\bar{s}$ are error mean and standard deviation of error, respectively.

Example 2. Let us consider a SDE

$$
\begin{aligned}
& d x(s)=-\frac{1}{6} s^{2} x(s) d s-\frac{1}{30} x(s) d B_{s}^{H} \\
& -\frac{1}{10(1-s)} x(s) d B_{1}(s)-\frac{1}{30} x(s) d B_{2}(s), \\
& \quad s \in(0, T), T<1, \\
& x(0)=\frac{1}{12},
\end{aligned}
$$

with the exact solution $x(t)=(1 / 12) \exp \left(-(1 / 30) B_{t}^{H}-\right.$ $(1 / 18) t^{3}-(1 / 1800) t^{2 H}-\int_{0}^{t}(1 / 10(1-s)) d B_{1}(s)-(1 / 30) B_{2}(t)-$ $(1 / 1800) t+(1 /(200(1-s))))$. The numerical results have been shown in Tables 4,5 , and 6 (with various Hurst parameters), where $\bar{x}$ and $\bar{s}$ are error mean and standard deviation of error, respectively.

\section{Conclusion}

This paper presents a numerical comparison between the approximation solution of the SDE driven by the FBM with Hurst parameter $H \in((1 / 2), 1)$ and $n$ independent one-dimensional SBM and the exact solution of it. Also, the method is applied with two examples to illustrate the accuracy and implementation of the method.

\section{Conflict of Interests}

The authors declare that there is no conflict of interests regarding the publication of this paper.

\section{Acknowledgments}

The authors thank Islamic Azad University for supporting this work. The authors are also grateful to the anonymous referee for his/her constructive comments and suggestions.

\section{References}

[1] J. Bertoin, "Sur une intégrale pour les processus à $\alpha$-variation bornée," The Annals of Probability, vol. 17, no. 4, pp. 1277-1699, 1989.

[2] P. Cheng and M. Webster, "Stability analysis of impulsive stochastic functional differential equations with delayed impulses via comparison principle and impulsive delay differential inequality," Abstract and Applied Analysis, vol. 2014, Article ID 710150, 2014.

[3] W. Gao, F. Deng, R. Zhang, and W. Liu, "Finite-time $H_{\infty}$ control for time-delayed stochastic systems with Markovian switching," Abstract and Applied Analysis, vol. 2014, Article ID 809290, 2014.

[4] J. Guerra and D. Nualart, "Stochastic differential equations driven by fractional Brownian motion and standard Brownian motion," Stochastic Analysis and Applications, vol. 26, no. 5, pp. 1053-1075, 2008.

[5] D. Huang and S. K. Nguang, "Robust $H_{\infty}$ static output feedback control of fuzzy systems: an ILMI approach," IEEE Transactions 
on Systems, Man, and Cybernetics B, vol. 36, no. 1, pp. 216-222, 2006.

[6] A. Mark, F. Yao, and M. Hua, "Abstract functional stochastic evolution equations driven by fractional Brownian motion," Abstract and Applied Analysis, vol. 2014, Article ID 516853, 2014.

[7] K. Maleknejad, M. Khodabin, and M. Rostami, "A numerical method for solving $m$-dimensional stochastic Itô-Volterra integral equations by stochastic operational matrix," Computers \& Mathematics with Applications, vol. 63, no. 1, pp. 133-143, 2012.

[8] K. Maleknejad, M. Khodabin, and M. Rostami, "Numerical solution of stochastic Volterra integral equations by a stochastic operational matrix based on block pulse functions," Mathematical and Computer Modelling, vol. 55, no. 3-4, pp. 791-800, 2012.

[9] J. Wang and K. Zhang, "Non-fragile $H_{\infty}$ control for stochastic systems with Markovian jumping parameters and random packet losses," Abstract and Applied Analysis, vol. 2014, Article ID 934134, 2014.

[10] H. Zhang, Y. Shi, and A. Saadat Mehr, "Robust static output feedback control and remote PID design for networked motor systems," IEEE Transactions on Industrial Electronics, vol. 58, no. 12, pp. 5396-5405, 2011.

[11] M. Zähle, "Integration with respect to fractal functions and stochastic calculus. I," Probability Theory and Related Fields, vol. 111, no. 3, pp. 333-374, 1998.

[12] L. Coutin, "An introduction to (stochastic) calculus with respect to fractional Brownian motion," in Séminaire de Probabilités XL, vol. 1899, pp. 3-65, Springer, Berlin, Germany, 2007.

[13] L. Decreusefond and A. S. Üstünel, "Fractional Brownian motion: theory and applications," in Systèmes Différentiels Fractionnaires, vol. 5 of ESAIM Proceedings, pp. 75-86, 1998.

[14] D. Nualart and A. Răşcanu, "Differential equations driven by fractional Brownian motion," Collectanea Mathematica, vol. 53, no. 1, pp. 55-81, 2002.

[15] H. Lisei and A. Soós, "Approximation of stochastic differential equations driven by fractional Brownian motion," in Seminar on Stochastic Analysis, Random Fields and Applications Progress in Probability, vol. 59, pp. 227-241, 2008.

[16] Y. Mishura and G. Shevchenko, "The rate of convergence for Euler approximations of solutions of stochastic differential equations driven by fractional Brownian motion," Stochastics, vol. 80, no. 5, pp. 489-511, 2008.

[17] F. Russo and P. Vallois, "Forward, backward and symmetric stochastic integration," Probability Theory and Related Fields, vol. 97, no. 3, pp. 403-421, 1993.

[18] F. Biagini, Y. Hu, B. Øksendal, and T. Zhang, Stochastic Calculus for Fractional Brownian Motion and Applications, Springer, London, UK, 2008.

[19] T. Caraballo, M. J. Garrido-Atienza, and T. Taniguchi, "The existence and exponential behavior of solutions to stochastic delay evolution equations with a fractional Brownian motion," Nonlinear Analysis. Theory, Methods \& Applications, vol. 74, no. 11, pp. 3671-3684, 2011.

[20] L. Longjin, F.-Y. Ren, and W.-Y. Qiu, “The application of fractional derivatives in stochastic models driven by fractional Brownian motion," Physica A, vol. 389, no. 21, pp. 4809-4818, 2010.

[21] B. Øksendal, Stochastic Differential Equations. An Introduction with Application, Springer, New York, NY, USA, 5th edition, 1998. 


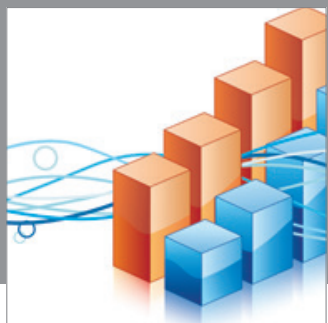

Advances in

Operations Research

mansans

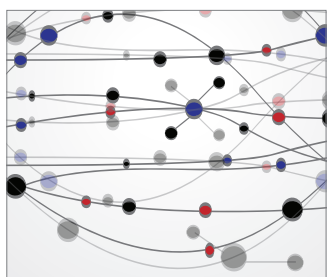

The Scientific World Journal
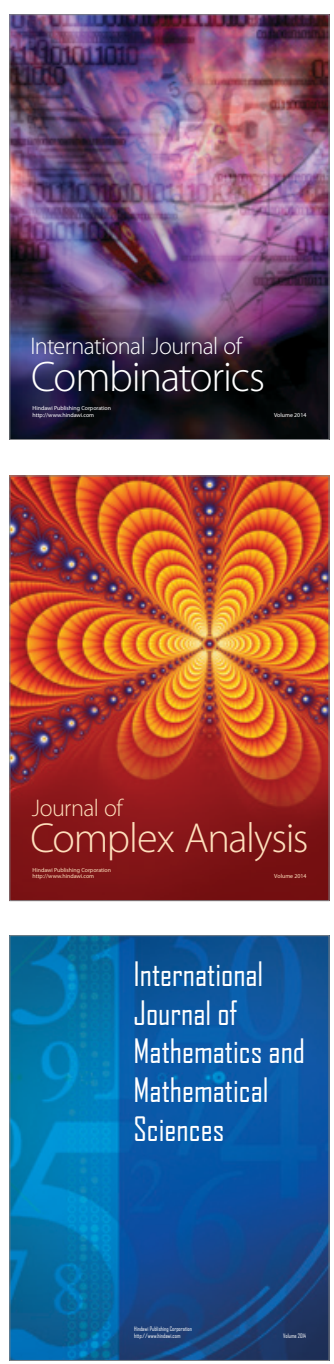
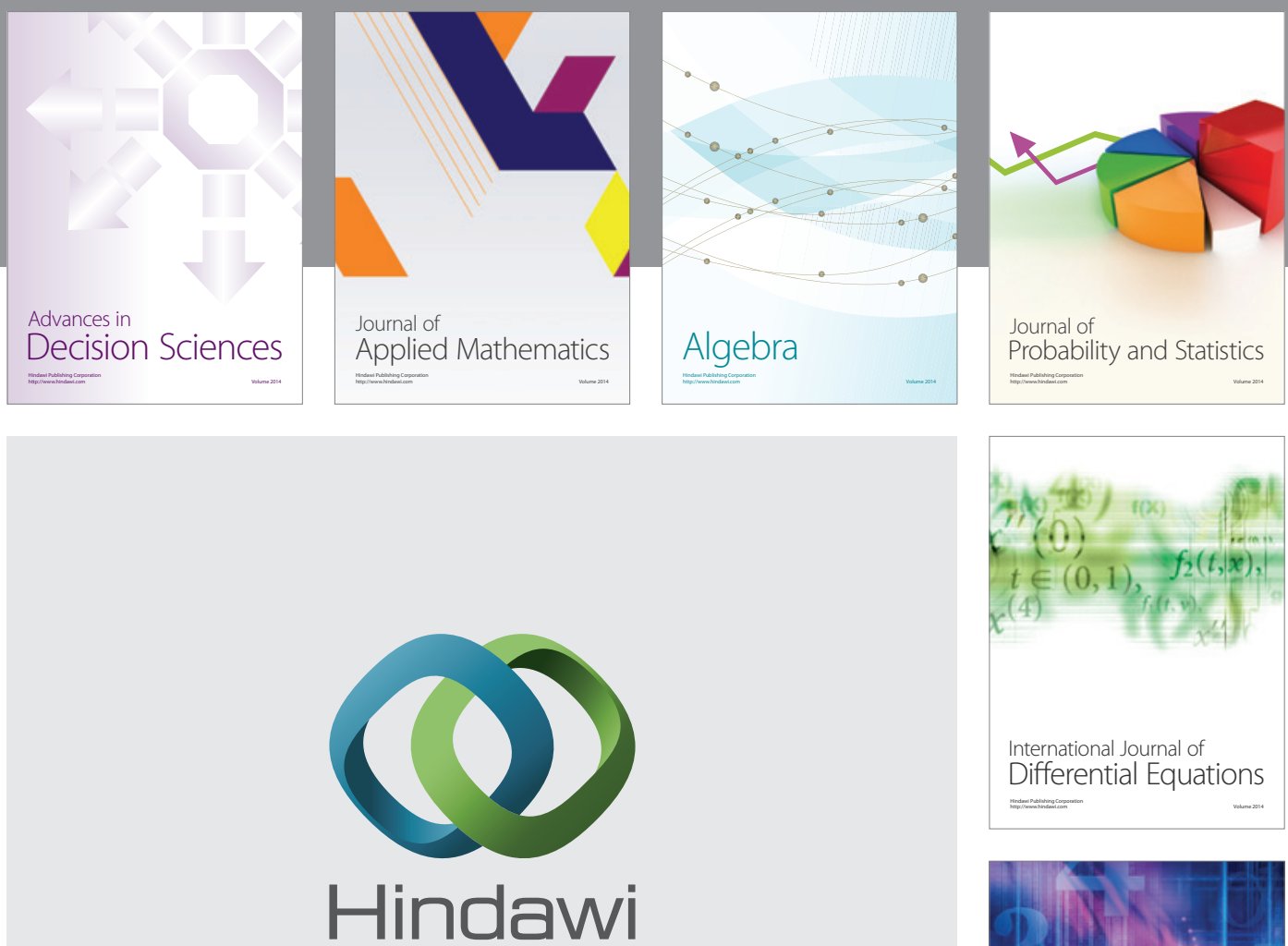

Submit your manuscripts at http://www.hindawi.com
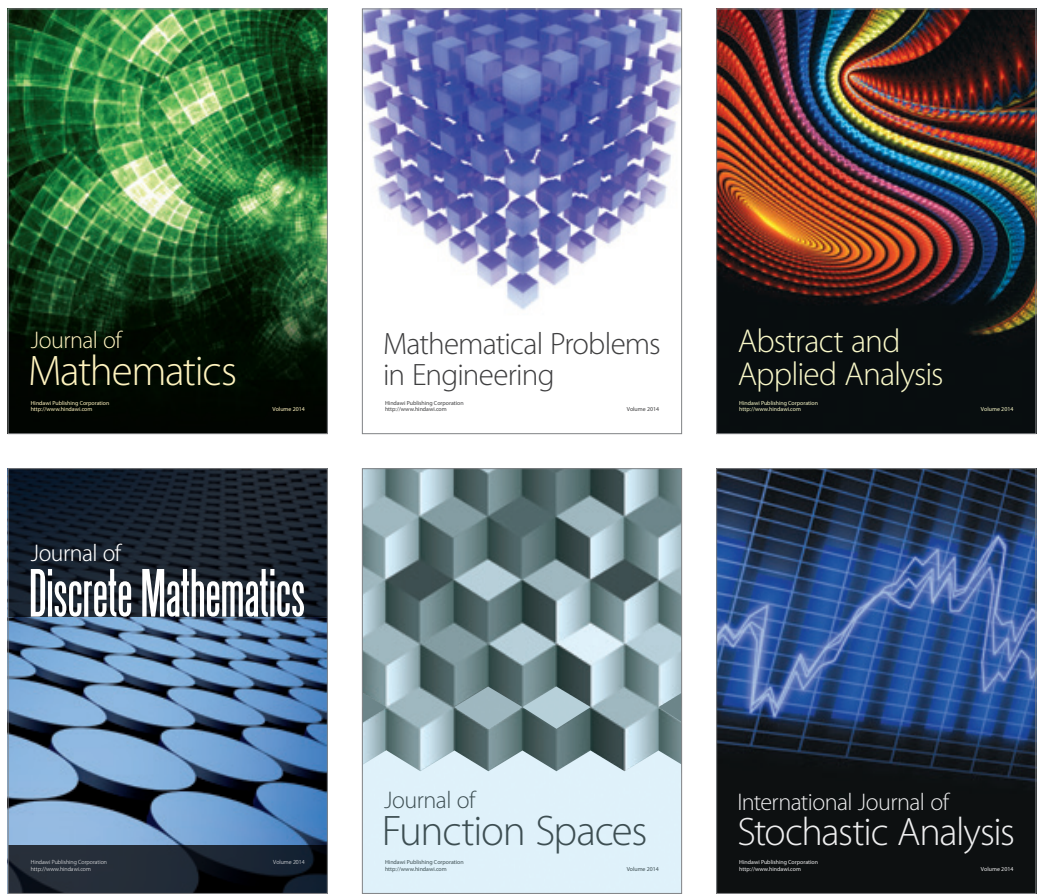

Journal of

Function Spaces

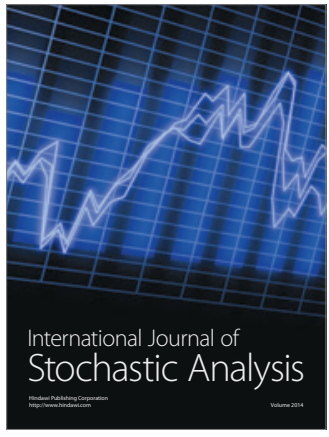

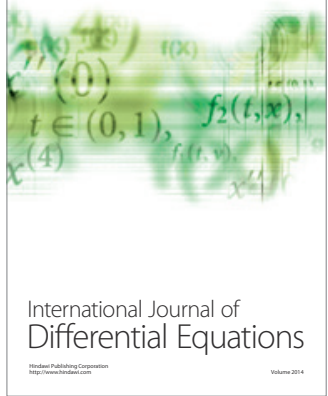
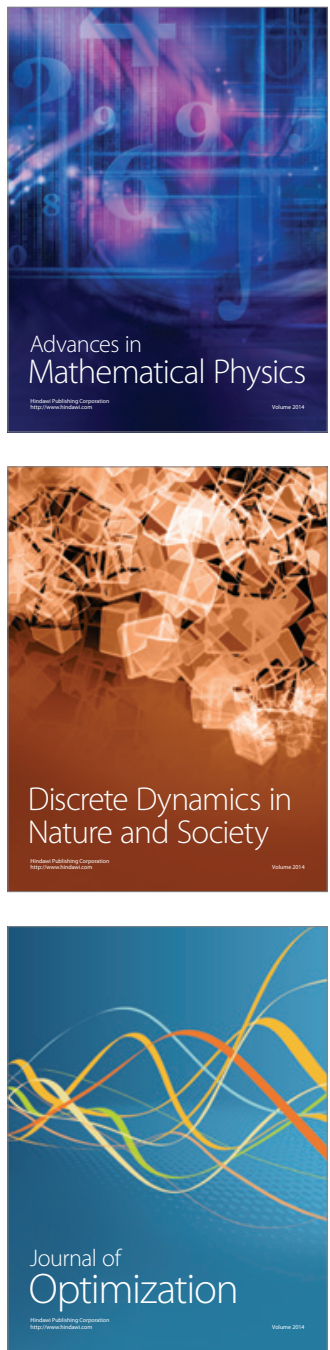